\title{
Privatisierung des Maßregelvollzugs in Schleswig-Holstein erlaubt
}

\author{
Artikel 20 Abs. 2, 33 Abs. 4 GG; § 3 Abs. 1 b MVollzG Schl.-H. \\ OLG Schleswig, Beschluss vom 19. 10. 2005 - 2W120 / 05
}

Heribert Ostendorf

\section{Sachverhalt}

$\mathrm{D}$ er Sachverhalt, der dem Beschluss des 2. Zivilsenats des Schleswig-Holsteinischen Oberlandesgerichts in Schleswig zugrunde lag, ist vertrackt. Formal ging es um die Eintragung einer privatrechtlichen Klinik $(\mathrm{GmbH})$ zur Durchführung des Maßregelvollzugs in das Handelsregister. Diese Eintragung war zunächst vom AG Flensburg (Beschluss vom 13.1.2005), sodann auf Beschwerde vom LG Flensburg (Beschluss vom 2.3.2005) abgelehnt worden. Das OLG Schleswig hat diese Beschlüsse aufgrund einer weiteren Beschwerde aufgehoben.

In der Sache geht es um die Privatisierung des Maßregelvollzugs. Bislang wurden die Maßregeln nach $\S 63$ Abs. 1 und $\S 64$ StGB sowie die einstweiligen Unterbringungen nach $\S 126$ a StPO gem. $\S 3$ Abs. 1 MVollzG Schleswig-Holstein a. F. von der Fachklinik Schleswig, einer rechtsfähigen Anstalt des Öffentlichen Rechts durchgeführt. Mit Gesetz zur Umwandlung psychiatrischer Einrichtungen und Entziehungsanstalten vom 24.9.2004 (Gesetz- und Verordnungsblatt Schleswig-Holstein S. 350) wurde die oberste Landesgesundheitsbehörde ermächtigt, durch Verordnung die Fachklinik Schleswig in eine Gesellschaft mit beschränkter Haftung umzuwandeln. Dies ist gem. § 1 Abs. 1 Landesverordnung über den Formwechsel und die Veräußerung der Fachklinik Schleswig vom 9.11.2004 (Gesetz- und Verordnungsblatt Schleswig-Holstein S. 426) geschehen. Daraufhin wurde die Fachklinik im Rahmen eines Vergabeverfahrens an einen ausgewählten Bewerber veräußert. Zugleich erging auf der Grundlage von $\S 3$ Abs. $1 \mathrm{~b}$ MVollzG n.F. i.V.m. § 24 Abs. 1 LVwG Schl.-H. ein Verwaltungsakt des zuständigen Ministeriums für Soziales, Gesundheit und Verbraucherschutz zur Beleihung der aus dem Formenwechsel der Fachklinik Schleswig (Anstalt des Öffentlichen Rechts) entstehenden Fachklinik Schleswig gGmbH mit der Aufgabe des Maßregelvollzugs (Amtsblatt Schleswig-Holstein S. 1072). Darin wurden dem Unternehmen die zur Erledigung der übertragenen Aufgabe erforderlichen hoheitlichen Befugnisse verliehen. Insbesondere wurde darin das Unternehmen unter Regelung des Beleihungsverhältnisses im Einzelnen ermächtigt, die Unterbringungen unter Freiheitsentzug durchzuführen und Zwangsmaßnahmen auf der Grundlage der $\S \S 6-13$ MVollzG anzuordnen und durchzuführen.

Der Formwechsel wird aber erst mit der Eintragung der Gesellschaft in das Handelsregister wirksam - insofern schließt sich der Kreis zur formalen Entscheidungsgrundlage.

\section{Gründe (gekürzt)}

Nach $\S 9$ c Abs. 1 und 2 GmbHG hat das Registergericht die Ordnungsgemäßheit der Errichtung und Anmeldung einer $\mathrm{GmbH}$ auch in materieller Hinsicht zu prüfen. Die Rechtmäßigkeitskontrolle hat sich darauf zu beziehen, dass die zwingenden gesellschaftsrechtlichen Anforderungen an die Gründung eingehalten und die notwendigen Gründungsakte nicht ganz oder teilweise wegen Verstoßes gegen gesetzliche Vorschriften nichtig sind. ...

Eine Nichtigkeit des Verwaltungsaktes lässt sich nicht feststellen, weil er - seine Rechtswidrigkeit unterstellt - jedenfalls nicht an einem besonders schwerwiegenden Fehler leidet, der bei verständiger Würdigung aller in Betracht kommenden Umstände offensichtlich ist, und auch sonst keine Nichtigkeitsgründe vorliegen ( $\$ 113$ Abs. 1 und 2 LVwG; vgl. auch BGH NJW 1979, 597; Kopp/ Ramsauer, VwVfG, 8. Aufl., § 44. Rn. 7 ff.). ...

Grundgesetz und Bundesgesetze lassen ein ausdrückliches Verbot, auf Grund eines Landesgesetzes durch Verwaltungsakt den Maßregelvollzug auf eine privatrechtlich verfasste Einrichtung als Aufgabe zur Erledigung in den Handlungsformen öffentlichen Rechts unter der Aufsicht des Landes zu übertragen (Funktionalprivatisierung im Wege der Beleihung), nicht erkennen. Das Bundesverfassungsgericht hat über die verfassungsrechtliche Zulässigkeit einer solchen oder einer vergleichbaren Maßnahme bisher nicht entschieden. Soweit andere Gerichte mit dieser Frage befasst waren, sind sie - allerdings ohne nähere Problematisierung - von der verfassungsrechtlichen Zulässigkeit ausgegangen (OLG Hamm, Beschluss vom 22.11.1979, 1 VAs 39/78 - Juris; KG ZfStrVo 2002, 248, 249 für den Fall einer - dort allerdings fehlenden - gesetzlichen Beleihungsgrundlage; Thü- ringer Verfassungsgerichtshof, Beschluss vom 23.10.2002, VerfGH 11/02 - unveröffentlicht). In der Literatur ist die Frage der verfassungsrechtlichen Zulässigkeit umstritten (vgl. Kammeier, Maßregelvollzugsrecht, 2. Aufl., Anm. A 130)....

Allerdings hat das Bundesverfassungsgericht in seinem Beschluss vom 5.12.2002 (E 107, 59, 91) Wasserverbandsfall - klargestellt, außerhalb der unmittelbaren Staatsverwaltung und der gemeindlichen Selbstverwaltung sei das Demokratiegebot des Art. 20 Abs. 2 GG offen für Formen der Organisation und Ausübung von Staatsgewalt, die vom Erfordernis lückenlosen personeller demokratischer Legitimation aller Entscheidungsbefugten abwichen. Es sei dann erforderlich, dass die Aufgaben und Handlungsbefugnisse der Organe in einem von der Volksvertretung beschlossenen Gesetz ausreichend vorherbestimmt seien und ihre Wahrnehmung der Aufsicht personell demokratisch legitimierter Amtswalter unterliege (vgl. ebenfalls für die funktionale Selbstverwaltung Jarass/Pieroth, GG, 7. Aufl., Art. 20 Rn. 10 a: Sommermann in v. Mangoldt/Klein/Stark, GG, 4. Aufl., 2. Bd., Art. 20 Abs. 2 Rn. 166). Eine unmittelbare Staatsverwaltung ist für den Maßregelvollzug gesetzlich nicht angeordnet. Nach § 138 Abs. 1 StVollzG richtet sich die Unterbringung in einem psychiatrischen Krankenhaus oder in einer Entziehungsanstalt grundsätzlich nach Landesrecht. In Schleswig-Holstein - wie in vielen anderen Bundesländern - wurde und wird dementsprechend der Maßregelvollzug (bisher), anders als gesetzlich zwingend der Strafvollzug (vgl. §§ 139, 156, 155 StVollzG), von den Fachkliniken als rechtsfähigen Anstalten des öffentlichen Rechts (§ 3 Abs. 2 FachklinikG vom 13.01.2003; § 41 LVwG) - als deren eigene Aufgaben vollzogen (§ 3 MVollzG). Eine danach in Betracht kommende zulässige Lockerung der Legitimation als Ausnahme für Beliehene (vgl. Sommermann a.a.O. Rn. 168 unter Hinweis auf Dreier, GG, II. Band, Art. 20 Rn. 108 und 115) erscheint jedenfalls nicht als von vornherein fernliegend. Dementsprechend hat der Bremer StGH die Legitimation der durch einen beliehenen Unternehmer ausgeübten öffentlichen Gewalt als grundsätzlich hinreichend gewährleistet angesehen (NVwZ 2003, 81, 83; vgl. auch OVG Lüneburg, Beschluss vom 21.7.1997, 7K 7532/95 - Juris). Danach führt hier hinsicht- 
lich der personellen Legitimation die Kette vom demokratisch gewählten Landesparlament über die Beleihungsermächtigung gemäß $§ 24$ Abs. 1 LVwG in Verbindung mit $\S 3$ Abs. 1 b MVollzG n.F. zu dem zuständigen Ministerium und von diesem durch den Verwaltungsakt an die gGmbH. Eine weitergehende personelle Legitimation ist darin zu sehen, dass die Bestellung des Leiters der forensischen Abteilung und seines Vertreters - jeweils eines Facharztes für Psychiatrie - der Einwilligung der Aufsichtsbehörde - der obersten Landesgesundheitsbehörde oder der durch Verordnung von ihr bestimmten Landesbehörde - bedarf, die wegen erheblicher Bedenken hinsichtlich der fachlichen Qualifikation oder der persönlichen Zuverlässigkeit der für die Leitung in Aussicht genommenen Person verweigert werden kann (Ziff. I 7 Verwaltungsakt). ...

Angesichts der beschriebenen Legitimation ist auch zweifelhaft, ob - wie Amts- und Landgericht meinen - die Privatisierung des Maßregelvollzuges durch Beleihung schon deswegen verfassungsrechtlich scheitert, weil die Angestellten der gGmbH oftmals sofort durchsetzbare Ermessungsentscheidungen bei hoher Grundrechtsrelevanz $\mathrm{zu}$ treffen haben werden, ohne dass eine stets "mitschreitende Kontrolle» durch einen Berufsbeamten erfolgt (so hinsichtlich der Privatisierung des Strafvollzuges Kruis ZRP 2000, 1, 4; wohl auch Di Fabio JZ 1999, 585, 592: Sicherung einer tatsächlichen Sachherrschaft für die Verwaltung in eingriffstypischen Handlungsfeldern, der Hinweis auf KG NJW 1997, 2894, 2896 betrifft allerdings keinen Beleihungsfall; Callies / Müller-Dietz, StVzG, 10. Aufl., Einl. Rn. 45; § 151 Rn. 1; i. E. a. A. Lange DÖV 2001, 898, 900). Im Hintergrund dieser Auffassung steht die Befürchtung, dass »nur der Eigennutz des Gefängnisunternehmers eine ausreichende Antriebskraft für die fortlaufend zu bewirkende ökonomische Optimierung der Organisation der Anstalt und ihres Betriebes sein kann « (Kruis a. a. O. S. 5); allein der Beamte sei auf Grund seiner Unabhängigkeit und Pflichteinstellung das optimale Exekutivorgan (Jachmann / Strauß ZBR 1999, 289, 292, 296; Manssen ZBR 1999, 255, 257). Dem kann entgegengehalten werden, dass im vorliegenden Privatisierungsfall das Land für die mit der Durchführung des Maßregelvollzuges verbundenen Personal-, Sach- und Investitionskosten aufkommt (Ziff. I 11 Abs. 1 Verwaltungsakt). Soweit der Beliehene oder einer seiner Angestellten öffentliche Gewalt ausübt, ist er Amtsträger im Sinne des $\S 11$ Abs. 1 Nr. 2 c StGB und dementsprechend strafrechtlich verantwortlich (Wolff/Bachof/Stober, Verwaltungsrecht II, 5. Aufl., § 104 Rn. 11). Zwar ist die Grundrechtsrelevanz im Maßregelvollzug mindestens genauso hoch wie im Strafvollzug (wobei freilich ebenso die Entziehung der Freiheit als solche auf bereits vorangegangener gerichtlicher Anordnung beruht). Anders jedoch als dort richtet sich die Behandlung der in einem psychiatrischen Krankenhaus Untergebrachten - dem Charakter dieser Institution entsprechend - nach ärztlichen Gesichtspunkten, und zwar denselben, die für die Behandlung der anderen Patienten solcher Anstalten gelten ( $\$ 136$ StVollzG; Calliess/Müller-Dietz a.a.O. § 136 Rn. 1; § 138 Rn. 1). Entscheidend kommt es mithin auf den Sachverstand der Fachärzte als hauptsächlicher Entscheidungsträger (vgl. insbesondere $§ \S 4$ bis 7 MVollzG) an. Es kann aber davon ausgegangen werden, dass Ärzte grundsätzlich nicht weniger pflichtbewusst und unabhängig handeln als Berufsbeamte. Unmittelbarer Zwang ( $\$ 239$ LVwG) zur Durchsetzung von Anordnungen nach dem MVollzG darf ohnehin nur von Vollzugsbeamten gemäß § 252 LVwG angewendet werden ( 8 MVollzG).

Der Senat weist zur Vermeidung nicht auszuschließender Missverständnisse vorsorglich darauf hin, dass der Beschluss nicht abschließend dazu Stellung nimmt, ob der Verwaltungsakt verfassungswidrig ist oder nicht. Wäre er verfassungswidrig, so ist er jedenfalls nicht nichtig. Über die Verfassungsmäßigkeit der funktionellen Privatisierung des Maßregelvollzuges im Wege der Beleihung werden die zuständigen Gerichte - letztlich das Bundesverfassungsgericht - in den dazu vorgesehenen Verfahren entscheiden müssen.

\section{Anmerkung}

Die »Privatisierungswelle« macht vor der Justiz keinen Stopp. In Hessen ist der Strafvollzug teilprivatisiert, die Bewährungs- und Gerichtshilfe ist in Baden-Württemberg einem privaten Träger überantwortet. In mehreren Bundesländern wird die Privatisierung des Maßregelvollzugs vorangetrieben. Vordergründig soll effizienter gearbeitet, hintergründig sollen Gelder eingespart werden. Auch gegen die zweite, die eigentliche Zielsetzung, ist selbstverständlich nichts einzuwenden wenn es nicht auf Kosten der Betroffenen geht, damit letztlich auch auf Kosten der Gesellschaft. Wird weniger eingesetzt zur Resozialisierung, zur Rehabilitation, wird die Rückfallgefahr nach Entlassung erhöht. Wenn die Entlassung hinausgeschoben wird nach dem Motto »wegsperren für immer «, verursacht ein solcher Verwahrvollzug mehr Kosten als ein kürzerer qualitativer Resozialisierungsvollzug. Das sind primär kriminalpolitische Erwägungen, aber mit einer rechtlichen Dimension.

Die Qualität des Straf- und Maßregelvollzugs ist von Verfassungs wegen geboten. Es gibt ein verfassungsrechtliches Resozialisierungsgebot: »Die Verfassung gebietet, den Strafvollzug auf das Ziel der Resozialisierung der Gefangenen hin auszurichten. Der einzelne Gefangene hat nach Art. 2 Abs. 1 i.V.m. Art. 1 Abs. 1 GG einen grundrechtlichen Anspruch darauf, dass dieser Zielsetzung bei ihn belastenen Maßnahmen genügt wird « (BVerfG NJW 1998, S. 3337). Dieser Resozialisierungsauftrag ist zugleich eine Konkretisierung der sozialstaatlichen Fürsorgepflicht (Art. 20 Abs. 1, 28 Abs. 1, 74 Abs. 1 Nr. 7 GG). Hinzu kommt die rechtsstaatliche Machtverantwortung des Staates bei Vollstreckung von strafjustiziell angeordneten Sanktionen. Wenn die dritte Gewalt im Staate, die Justiz, Strafsanktionen verhängt, die zu gravierenden Grundrechtseinschränkungen führen, wie im Straf- und Maßregelvollzug, so bleibt der Staat auch in der Verantwortung für die Vollstreckung dieser Sanktionen, d. h. er muss Verantwortung für die Vollstreckung dieser Sanktionen, d. h. er muss für die korrekte Umsetzung dieser Sanktionen sorgen und Missbräuchen entgegenwirken. Der Staat darf diese Verantwortung nicht delegieren. ${ }^{1}$ Diesen grundrechtlichen Anforderungen wird die Entscheidung des 2. Zivilsenates nicht gerecht, auch wenn über die Verfassungswidrigkeit bzw. Verfassungsgemäßheit nicht abschließend entschieden wurde. Insbesondere wird der Machtunterworfenheit in der »totalen Institution « des Maßregelvollzuges zu wenig Bedeutung zugemessen. Diese Machtunterworfenheit drückt sich insbesondere in der Ermächtigung aus, Unmittelbaren Zwang auszuüben, Eingriffe in das Brief-, Post- und Fernmeldegeheimnis vorzunehmen, Besuche zu untersagen sowie besondere Sicherungsmaßnahmen wie Fixierung oder Fesselung, Einzeleinschließung und Ruhigstellung durch Medikamente durchzuführen. Wenn demgegenüber in dem Beschluss betont wird, dass unmittelbarer Zwang nur von Vollzugsbeamten gem. § $252 \mathrm{LVwG}$ angewendet werden darf, wird übersehen, dass der private Betreiber mit dem Beleihungsakt ausdrücklich hierzu ermächtigt worden ist, was gem. § 252 Abs. 1 Nr. 2 LVwG auch grundsätzlich gestattet ist. Damit ist ein wesentlicher Argumentationspfeiler des Beschlusses weggebrochen.

Der Alltag der Maßregelpatienten wird nicht allein vom ärztlichen Ethos bestimmt, sondern auch und vor allem von den organisatorischen Rahmenbedingungen, von der personellen und therapeutischen Ausstattung und vor allem auch von der Bereitschaft, verantwortbare Risiken für die Wiedereingliederung zu übernehmen. In Übereinstimmung mit den Entscheidungen des Amtsgerichts und Landgerichts Flensburg spricht mehr für die Verfassungswidrigkeit der Privatisierung des Maßregelvollzuges in Schleswig-Holstein als für die Verfassungsgemäßheit.

\section{Der Verfasser ist Mitherausgeber dieser Zeitschrift.}

\section{Fußnote}

1 S. Callies / Müller-Dietz, StVollzG, 10. Aufl., Einl. Rn. 45; Böhm, Strafvollzug, 3. Aufl., Rn. 87; Laubenthal, Strafvollzug, 3. Aufl., Rn. 45; Kaiser in: Strafvollzug, hrsg. von Kaiser / Schöch, 5. Aufl., § 4 Rn. 30 ff.; Wagner ZRP 2000, 172; Gusy/Lührmann StV 2001, 47; Bonk JZ 2000, 441; Di Fabio JZ 1999, 591; Kruis ZRP 2000, 2 ff.; weitergehend Kulas, Privatisierung hoheitlicher Verwaltung/Zur Zulässigkeit privater Strafvollzugsanstalten, 1996, S. 139: Privatisierung von etwa $15-20 \%$ der insgesamt in der Bundesrepublik zur Verfügung stehenden Haftplätze erlaubt; ebenso Müther in: Privatisierung des Haftvollzugs und Kriminalpolitik in Europa, hrsg. von Herrfahrdt, 2005, S. 18. 